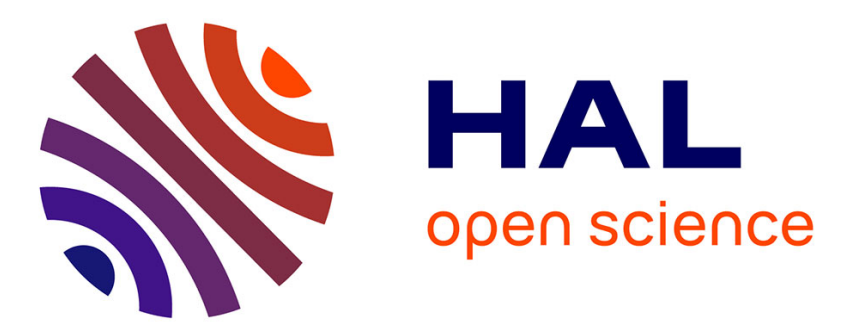

\title{
Energy localization effects within a reverberation chamber and their reduction in chaotic geometries
}

Kamardine Selemani, Elodie Richalot, Olivier Legrand, Odile Picon, Fabrice Mortessagne

\section{- To cite this version:}

Kamardine Selemani, Elodie Richalot, Olivier Legrand, Odile Picon, Fabrice Mortessagne. Energy localization effects within a reverberation chamber and their reduction in chaotic geometries. IEEE Transactions on Electromagnetic Compatibility, 2017, 59 (2), pp.325. 10.1109/TEMC.2016.2617322 . hal-01393157

\section{HAL Id: hal-01393157 \\ https://hal.science/hal-01393157}

Submitted on 16 Jan 2017

HAL is a multi-disciplinary open access archive for the deposit and dissemination of scientific research documents, whether they are published or not. The documents may come from teaching and research institutions in France or abroad, or from public or private research centers.
L'archive ouverte pluridisciplinaire HAL, est destinée au dépôt et à la diffusion de documents scientifiques de niveau recherche, publiés ou non, émanant des établissements d'enseignement et de recherche français ou étrangers, des laboratoires publics ou privés. 


\title{
Energy localization effects within a reverberation chamber and their reduction in chaotic geometries
}

\author{
K. Selemani, E. Richalot, O. Legrand, O. Picon, F. Mortessagne
}

\begin{abstract}
A method of studying energy localization effects in a reverberation chamber is proposed and applied to simulation results. Its application to field spatial distributions of a classical reverberation chamber equipped with a mode stirrer shows a strong energy localization phenomenon of damaging effect on the field homogeneity. From the energy distributions it appears that this phenomenon is principally related to energy localization around the mode stirrer. To avoid the presence of a mode stirrer causing this phenomenon, an alternative shape of reverberation chamber, inspired from geometries of chaotic cavities, is proposed. The results obtained with this new cavity configuration show that the strong energy localizations are avoided and the resonant modes better contribute to the field uniformity and isotropy within the working volume.
\end{abstract}

Index Terms-Chaotic cavity, field homogeneity, field localization, reverberation chamber, statistical distribution.

\section{INTRODUCTION}

$\mathbf{I}$ $\mathrm{N}$ electromagnetic compatibility (EMC) tests using a reverberation chamber (RC), the device under test (DUT) is placed within the so-called working volume [1], situated in the cavity central volume, in which a uniform and isotropic field distribution is required. Since only the field in the working volume is useful to illuminate the DUT, it is important to optimize the power transmission towards this area, in particular when high field levels are required to test the device immunity. The understanding of wave function structure in complex geometries where the ray dynamics is nonintegrable has been a major interest of Wave Chaos research. Very early, the existence of large fluctuations in wave function intensity, with a large excess of intensity near the short unstable periodic orbits, was observed [2] [3] [4] and coined scarring. In chaotic cavities, this phenomenon was clearly identified as the main nonrandom features of chaotic wave functions. In systems where the dynamics of rays are not fully chaotic, wavefunctions may display localization effects related to trapping on regular islands [5].

Due to the strongly scattering nature of a classical mode stirrer and the multiple scattering environment created by the resonant cavity, such a phenomenon is likely to appear in an RC. The large dimensions of the stirrer in terms of the wavelengths under consideration precludes the appearance of strong localization effects, but a weak field enhancement

This work was supported by the French National Research Agency (ANR) under the project CAOREV. K. Selemani is with CMN Cherbourg, 51 rue de la Bretonnière, 50105 Cherbourg Cedex, France (kselemani@cmncherbourg.com). E. Richalot and O. Picon are with the Université ParisEst, ESYCOM (EA 2552), UPEMLV, ESIEE-Paris, CNAM, F-77454 Marnela-Vallée, France (elodie.richalot, odile.picon)@univ-mlv.fr. O. Legrand and F. Mortessagne are with the LPMC, CNRS UMR 7336, Université de Nice-Sophia Antipolis, F-06108 Nice Cedex 2, France (olivier.legrand, fabrice.mortessagne)@unice.fr. can be envisaged. In this case, the field would no longer be homogeneous over the whole cavity, even if the homogeneity could still be observed in a restricted zone. For this reason, we investigate in this paper the field localization within an RC equipped with a mode stirrer, and propose a new cavity shape designed to avoid such a phenomenon, where the key ingredient is to replace the classical stirrer by a moving metallic hemisphere. The local field enhancement phenomenon is brought to light in two ways. Our first approach consists in calculating an indicator of the existence of an electric or magnetic energy enhancement area in the total volume of both cavities; it is called the Inverse Partition Ratio and has been widely used to quantify wave function localization either in chaotic or disordered wave systems [6] [7] [8]. In our second approach, dedicated to the research of the field enhancement areas, we divide each cavity into sub-volumes, and compare the mean energies in the different sub-volumes. We will see that the localization effect is principally due to the presence of the mode stirrer and restricted to its vicinities, and is drastically reduced in our proposed alternative $\mathrm{RC}$ shape. Both approaches are applied at a fixed position of the stirrer and hemipshere as well as for different positions of them over a whole rotation.

It will be shown how such a field localization phenomenon affects the field statistical uniformity and isotropy. As a consequence, an improvement of the field properties is obtained when this phenomenon is avoided.

The improvement of the RC behavior with the suggested cavity shape modifications is finally shown with regard to standardized criteria from the norm governing EMC test in reverberation chambers [1].

\section{STUdiED MICROWAVE CAVITIES}

In order to illustrate simply the appearance of a localization phenomenon of electric or magnetic energy in a reverberant room, we consider in the first instance a $2 \mathrm{D}$ rectangular cavity provided with a metallic mode stirrer of complex shape (Fig. 1). The cavity is of length $756 \mathrm{~mm}$ and width $456 \mathrm{~mm}$, walls and stirrer are of perfect metal. Using HFSS software for electromagnetic simulations, the eigenmodes of the cavity have been determined. Fig.1 shows the distribution of electric and magnetic field amplitudes for the $53^{r d}$ eigenmode at 2.3873 $\mathrm{GHz}$. We observe higher energy densities in the central area of the cavity (the useful zone), wherein the DUT could be placed. Thus this mode will contribute to achieve the required energy level in the working area. Indeed, the mean electric and magnetic energies calculated within the working area are higher than the ones calculated in the rest of the cavity volume: 


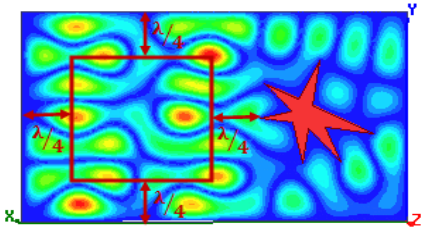

(a) Electric field amplitude

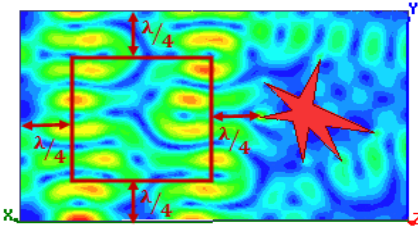

(b) Magnetic field amplitude Fig. 1. Normalized amplitudes of electric and magnetic fields at $f=$ $2.3873 \mathrm{GHz}$ for a 2D cavity with a metallic stirrer (in red). The red line indicates the working area situated at a quarter-wavelength from the walls and stirrer.

the ratio of the mean energies calculated in the working volume and outside is of 3.42 for the electric field and 3.03 for the magnetic one.

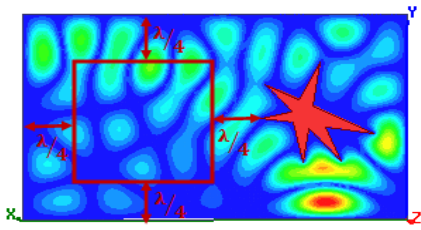

(a) Electric field amplitude

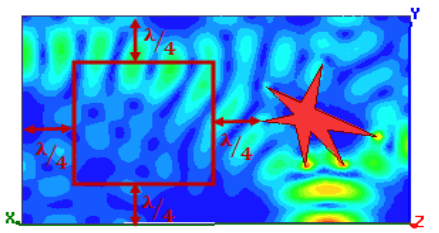

(b) Magnetic field amplitude
Fig. 2. Normalized electric and magnetic field amplitudes at $f=$ $2.2087 \mathrm{GHz}$ for a 2D cavity with a metallic stirrer (in red).

A different behavior is observed with the $44^{\text {th }}$ eigenmode at $2.2087 \mathrm{GHz}$ depicted in Fig.2. In this case, the electric and magnetic energy densities are higher in the area situated between the mode stirrer and one of the closest walls, so that the effect of this mode in the working area is reduced. In this case, the ratio of the mean energies calculated in the working volume and outside is of 0.655 for the electric field and 0.608 for the magnetic one.

This simple case highlights an energy localization phenomenon in a reverberation cavity disturbed by a mode stirrer. To examine its effect on the functioning of a reverberation chamber, this phenomenon will now be examined in 3D cavities and the effect of the mode stirrer displacement will be taken into acount.

Two parallelepipedic cavities of dimensions $\mathrm{W}=0.785 \mathrm{~m}$ along (Ox), $\mathrm{L}=0.985 \mathrm{~m}$ along (Oy) and $\mathrm{H}=0.995 \mathrm{~m}$ along $(\mathrm{Oz})$ are considered (Fig.3). The classical RC (C1, Fig.3a) consists of a parallelepipedic cavity equipped with a mode stirrer. The shape and location of the latter conform to an industrial RC, except for a global scaling factor. The metallic paddles are $3 \mathrm{~mm}$ thick whereas the vertical circular rod at the panels rotation axis has a diameter of $8 \mathrm{~mm}$. All surfaces are considered as perfectly conducting.

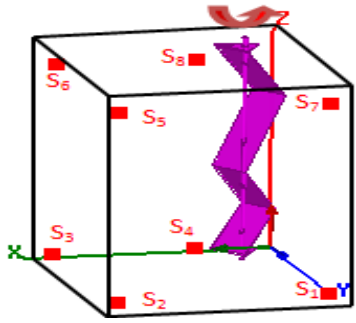

(a) Classical RC (C1)

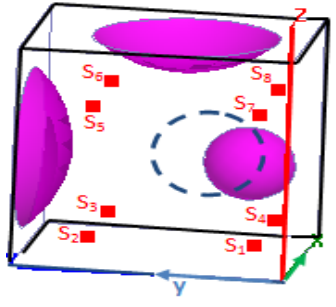

(b) Chaotic cavity (C2)
Fig. 3. Chaotic and classical cavities with 8 points sources $S_{i}$.
The previous $2 \mathrm{D}$ example suggests that the presence of a mode stirrer within the cavity volume could induce a field localization in its vicinity. Therefore we propose an alternative RC geometry with a hemispheric mode stirrer located on a cavity wall (C2, Fig.3b). Apart from avoiding localization effects, the chosen geometry also has to lead to field uniformity and isotropy. As in chaotic cavities most of the resonant modes present uniform and isotropic field distributions [9] [10] [11], we draw inspiration from an already studied 2D chaotic cavity [12] to propose a new 3D RC geometry. It consists of a parallelepipedic cavity provided with two metallic spherical caps and one metallic spherical hemisphere on its walls and in electrical contact with them, a spherical cap being a portion of a sphere cut off by a plane and whose height is, in our case, less than the sphere radius for a cap or equal for a hemisphere. In previous works we already studied this resonant cavity and showed its chaotic behaviour (with spatial and spectral statistics in agreement with those predicted by Random Matrix Theory [13]) as well as the improvement of the field properties with regard to the EMC standards in comparison to a classical RC [9] [14] [15]. The hemisphere has a radius of $15 \mathrm{~cm}$ and caps of $45 \mathrm{~cm}$ and $50 \mathrm{~cm}$. The highest penetration depth of the caps within the cavity is of $15 \mathrm{~cm}$. Whereas the two caps are fixed, the stirring process is ensured by the translation of the hemisphere. Its center rotates on the $\mathrm{x}=\mathrm{W}$ plane along a circle of radius $27.85 \mathrm{~cm}$ and centered at (W; L/2.1; H/2). The initial position of the hemisphere center, used in the following while studying the cavity in a fixed configuration, is (W; 25.5 $\mathrm{cm} ; 32 \mathrm{~cm}$ ).

The two cavities are simulated, without considering any excitation antenna, by using the eigenmode solver of HFSS software. Simulations are performed without considering any losses in the cavity, so that the electric field components are real. For both cavities, simulations are first of all performed for a fixed position of the stirrer/hemisphere, then for a whole stirrer/hemisphere rotation.

\section{EIGENMODES PROPERTIES}

\section{A. For a fixed stirrer position}

Originally proposed in quantum mechanics to distinguish the energy regions of localized states of a particle [16] [17], the Inverse Partition Ratio (IPR) has also been used in microwave cavities to evaluate the degree of localization of the electric field [18]. This indicator is calculated as :

$$
L_{k}^{A}=\frac{\iiint_{V}\left|A_{k}\right|^{4} d v}{\left[\iiint_{V}\left|A_{k}\right|^{2} d v\right]^{2}} \cdot V
$$

with $k=x, y$ or $z, A$ is the complex amplitude of electric or magnetic field.

For a real field component following a normal distribution this indicator is equal to $3(4.77 \mathrm{~dB})$, whereas it increases with a localization phenomenon. A global indicator of the field localization is calculated as the quadratic mean of the three IPR related to field components :

$$
L_{t o t}^{(E, H)}=\sqrt{\frac{\left(L_{x}^{(E, H)}\right)^{2}+\left(L_{y}^{(E, H)}\right)^{2}+\left(L_{z}^{(E, H)}\right)^{2}}{3}}
$$


Figure 4 shows the IPR of three rectangular electric field components as well as the global ones for the 330 first eigenmodes respectively in the chaotic cavity and in the classical cavity. We observe much higher IPR values attained in the classical RC compared to the chaotic one, the IPR values remaining close to the ideal value in the latter. It indicates a strong localization of the electric energy for many eigenmodes in C1. IPR values are particularly high at low frequencies, with numerous peaks above $20 \mathrm{~dB}$ below $0.564 \mathrm{GHz}$ (corresponding to the $42^{\text {nd }}$ mode), but the localization effect remains at high frequencies with peaks above $15 \mathrm{~dB}$ even after the $300^{\text {th }}$ mode $(1.08 \mathrm{GHz})$. In the chaotic cavity (Fig.4b), the maximal total IPR value is of $10 \mathrm{~dB}$ (only attained once) and IPR values remain below $6 \mathrm{~dB}$ after the $32^{\text {nd }}$ mode $(0.541 \mathrm{GHz})$. As a comparison, the global IPR index exceeds $10 \mathrm{~dB}$ for 86 modes in the classical cavity.

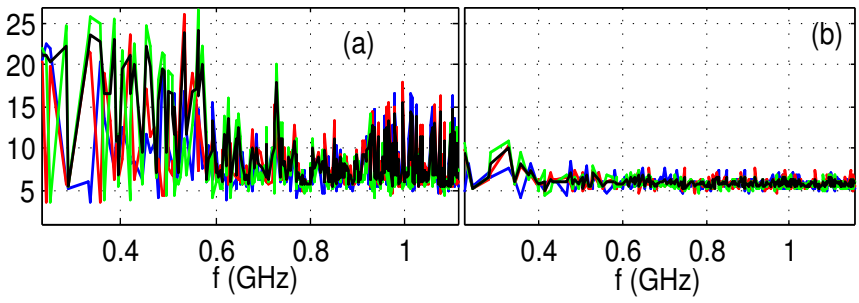

Fig. 4. Electric field localization index (dB), (a) in the classical RC, (b) in the chaotic cavity (blue for $\mathrm{x}$-, red for $\mathrm{y}$ - and green for $\mathrm{z}$-component and black for $\mathrm{L}_{t o t}^{E}$ ).

Thus it clearly appears that numerous modes of the classical RC undergo a strong electric energy localization; these eigenmodes present a heterogeneous distribution. In order to better quantify this phenomenon, Table I presents the means and standard deviations over the whole studied frequency band of the four examined indicators calculated from electric field distribution in each cavity. Due to the improvement of the energy uniformity with the frequency, these quantities are given while considering the 330 first modes then after excluding the first 30 and 60 ones. The resonant frequencies of the $30^{t h}$ and $60^{t h}$ modes are of $0.507 \mathrm{GHz}$ and $0.632 \mathrm{GHz}$ in $\mathrm{C} 1$, and $0.527 \mathrm{GHz}$ and $0.666 \mathrm{GHz}$ in $\mathrm{C} 2$.

\begin{tabular}{|c|c|c|c|c|c|c|c|c|}
\hline \multicolumn{9}{|c|}{ TABLE I } \\
\hline$L_{k}^{E}$ & \multicolumn{2}{|c|}{$L_{x}^{E}$} & \multicolumn{2}{|c|}{$L_{y}^{E}$} & \multicolumn{2}{|c|}{$L_{z}^{E}$} & \multicolumn{2}{|c|}{$L_{\text {tot }}^{E}$} \\
\hline & $\mu$ & $\sigma$ & $\mu$ & $\sigma$ & $\mu$ & $\sigma$ & & $\sigma$ \\
\hline C1 & 9.41 & 17.6 & 11.95 & 30.19 & 18.15 & 53.61 & 16.67 & 35.69 \\
\hline $\mathrm{C} 2$ & 3.87 & 0.58 & 3.9 & 0.73 & 3.89 & 0.87 & 3.91 & 0.59 \\
\hline \multicolumn{9}{|c|}{ without the first 30 modes } \\
\hline $\mathrm{Cl}$ & 7.27 & 7.70 & 9.34 & 25.06 & 8.84 & 29.37 & 10.11 & 22.07 \\
\hline $\mathrm{C} 2$ & 3.83 & 0.47 & 3.82 & 0.47 & 3.79 & 0.41 & 3.82 & 0.30 \\
\hline \multicolumn{9}{|c|}{ without the first 60 modes } \\
\hline $\mathrm{Cl}$ & 6.62 & 6.79 & 7.29 & 6.80 & 5.97 & 7.02 & 7.41 & 6.21 \\
\hline $\mathrm{C} 2$ & 3.82 & 0.45 & 3.80 & 0.45 & 3.75 & 0.35 & 3.80 & 0.26 \\
\hline
\end{tabular}

Table I confirms the previous observations. The mean IPR values close to the ideal one combined to their low frequential variation in the case of the chaotic cavity indicate a spatial and spectral uniformity of the electric field distribution. The much higher values of the means and standard deviations in the classical $\mathrm{RC}$ reflect the strong localization phenomenon suffered by numerous modes. By suppressing the first 30 then 60 modes, the standard deviations decrease in the chaotic cavity, whereas the means and standard deviations improve in the classical cavity. The performances of the chaotic cavity still remain far better than these of the classical RC.

So far, only the electric energy distribution has been considered. To verify that the observation of the magnetic energy distribution would lead to the same conclusions, the same indicators have been calculated using the IPR definition based on the magnetic field component amplitudes (Eq.1). A similar variation of both indicators, based on electric or magnetic fields, is observed. This similar behavior will allow us later on to restrict our study to the electric field distribution.

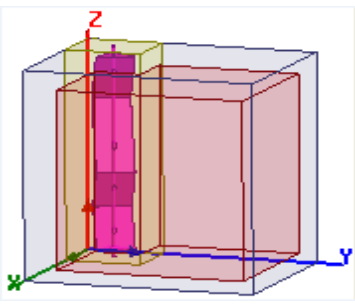

(a)

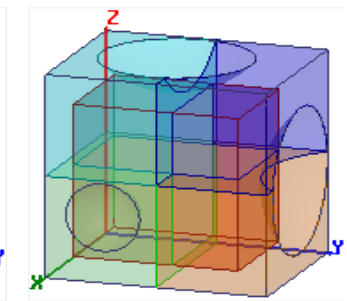

(b)
Fig. 5. Integration volumes (a) within the classical RC : volumes $V_{b}$ and $V_{z}$, and (b) within the chaotic cavity: peripheral volumes $\left(V_{1}, V_{2}, V_{3}, V_{4}\right)$ and central volume $V_{c}$.

We have highlighted a localization phenomenon in the classical RC, but IPR index doesn't indicate the region of higher energy density. To answer this question, we divide the studied cavities into several non-overlapping sub-volumes and compare their energy densities. In the classical RC (Fig.5a), a volume is defined around the stirrer (of size $27.2 \mathrm{~cm} \times 32.2 \mathrm{~cm}$ along $\mathrm{x}$ - and $\mathrm{y}$-axes) and another in the central area (at $50 \mathrm{~mm}$ from the cavity walls). The chaotic cavity is subdivided into four peripheral domains and a central one (at $50 \mathrm{~mm}$ from the cavity walls and caps) (Fig.5b). We then evaluate the ratio between the mean electric or magnetic energy densities within the volume around the stirrer $V_{b}$ and the central volume $V_{c}$ in the classical cavity and the ratios between the mean energy densities within each peripheral volume $V_{i}$ and the central volume $V_{c}$ in the chaotic cavity.

$$
R_{i}^{A}=\frac{\iiint_{V_{i}}|A|^{2} d v}{V_{i}} \cdot \frac{V_{c}}{\iiint_{V_{c}}|A|^{2} d v}
$$

with $V_{i}$ the peripheral volume ( $V_{b}$ in the conventional RC), $V_{c}$ the central volume, and $A$ the vectorial complex amplitude of electric or magnetic field. A uniform field distribution corresponds to a unit energy ratio, whereas a deviation from unity indicates a higher energy concentration in one of the defined volumes.

Fig. 6a representing the ratio of the mean energy around the stirrer and the one in the central volume of the conventional cavity shows a wide discrepancy with the ideal value, with numerous high ratio values (57 peaks of $R_{b}^{E}$ among 475 over 2). In contrast, in Fig. 6b, showing the ratios of mean electric energies in the four peripheral volumes of the chaotic cavity and the one in the central volume, all the curves remain close to unity on the whole frequency band, with only three peaks over 2; it indicates a uniform energy distribution between the five defined sub-domains. 


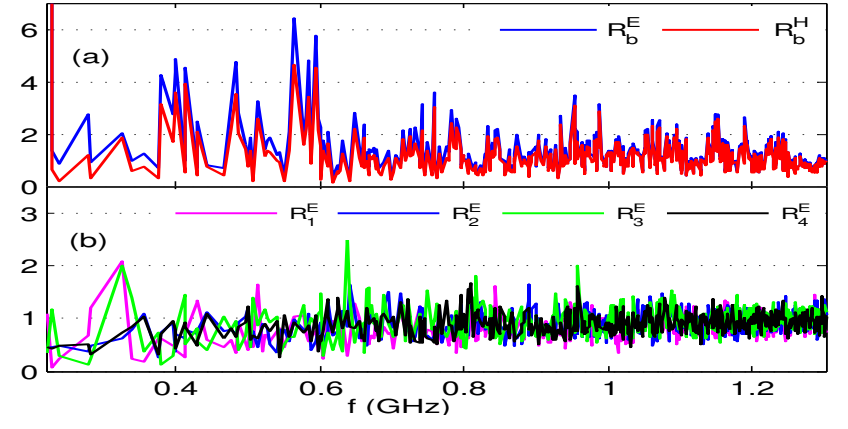

Fig. 6. Mean electric energy ratios for $R_{i}^{E}$ in (a) $\mathrm{C} 1$, (b) $\mathrm{C} 2$.

As already observed with the IPR index, the comparison of the ratios calculated using the electric and magnetic energies (Fig. 6a) shows similar behaviors, so that the study can be restricted to the observation of only one of them. We have chosen to focus on the electric energy distribution.

TABLE II
\begin{tabular}{|c|c||c|c|c|c|}
\hline Cavities & $\begin{array}{c}\text { Classical } \\
\text { RC }\end{array}$ & \multicolumn{5}{c|}{ Chaotic RC } \\
\hline Ratios & $\mathrm{R}_{b}$ & $\mathrm{R}_{1}$ & $\mathrm{R}_{2}$ & $\mathrm{R}_{3}$ & $\mathrm{R}_{4}$ \\
\hline$\left\langle R_{i}^{E}\right\rangle$ & 1.24 & 0.836 & 0.902 & 0.936 & 0.905 \\
\hline$\sigma\left(R_{i}^{E}\right)$ & 2.60 & 0.221 & 0.213 & 0.264 & 0.264 \\
\hline \multicolumn{7}{|c|}{ Without the first 30 modes } \\
\hline$\left\langle R_{i}^{E}\right\rangle$ & 1.10 & 0.845 & 0.915 & 0.947 & 0.918 \\
\hline$\sigma\left(R_{i}^{E}\right)$ & 0.533 & 0.193 & 0.206 & 0.243 & 0.200 \\
\hline \multicolumn{7}{|c|}{ Without the first 60 modes } \\
\hline$\left\langle R_{i}^{E}\right\rangle$ & 1.08 & 0.853 & 0.923 & 0.952 & 0.922 \\
\hline$\sigma\left(R_{i}^{E}\right)$ & 0.457 & 0.187 & 0.199 & 0.219 & 0.194 \\
\hline
\end{tabular}
Means and standard deviations of electric energy ratios in both

Table II summarizes the properties of the mean electric energy ratios in both cavities, either giving their mean and standard deviations on the whole frequency band or by excluding 30 and 60 modes. Whereas the means of the ratios are close to unity in the chaotic cavity, they are higher in the classical RC indicating an energy localization around the stirrer at the expense of the energy level required for EMC tests in the working area. In the chaotic cavity the ratios display low variation versus frequency, thus most of the modes ensure a uniform energy distribution between the five sub-volumes. On the contrary, the higher values of the ratio standard deviations in the conventional $\mathrm{RC}$ denote the spectral variability of the field spatial distribution properties.

As expected, all the mean values come closer to one and all the standard deviations decrease when the first 30 then 60 modes are excluded, but the performances of the chaotic RC remain better than those of the conventional RC.

To illustrate this phenomenon of energy localization around the mode stirrer, Fig. 7 depicts electric field amplitudes of a localized mode, namely the $205^{\text {th }}$ one $(956 \mathrm{MHz}$, $L_{\text {tot }}^{E}=16.238, L_{\text {tot }}^{H}=22.211, R_{b}^{E}=2.847, R_{b}^{H}=3.119$ ). The localization of the electric energies around the mode stirrer is obvious. The field amplitudes are low within the cavity working volume, so that this mode contributes very little to the illumination of the DUT. It is also to notice that IPR and energy ratios are in agreement with field distributions, as they all indicate that energies are localized.

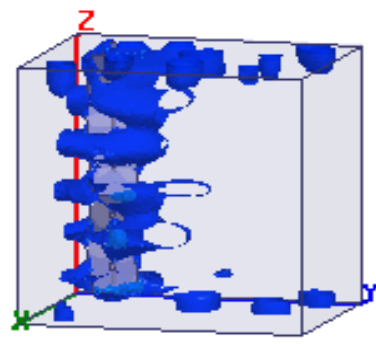

Fig. 7. Electric field amplitude of the $205^{\text {th }}$ mode ( $\mathrm{f}=908 \mathrm{MHz}$ ) represented by iso-amplitude surfaces. Only the amplitudes above an arbitrarily fixed limit are represented.

The comparison of IPR and energy ratios for all the modes of the conventional RC shows that over the 21 modes presenting $L_{\text {tot }}^{E}$ value larger than $12 \mathrm{~dB}$, only 4 are associated to $R_{b}^{E}$ ratio less than 1 (or $0 \mathrm{~dB}$ ). It shows the strong energy localization mainly occurs in the stirrer area.

\section{B. Effect of the stirrer rotation}

In this section, we evaluate the previously introduced indicators of localization in the two cavities for different positions of the stirrer and the hemisphere. This study aims to verify that the localization phenomenon observed for fixed cavity geometries is not bounded to a specific configuration but occurs at several stirrer or hemisphere positions.

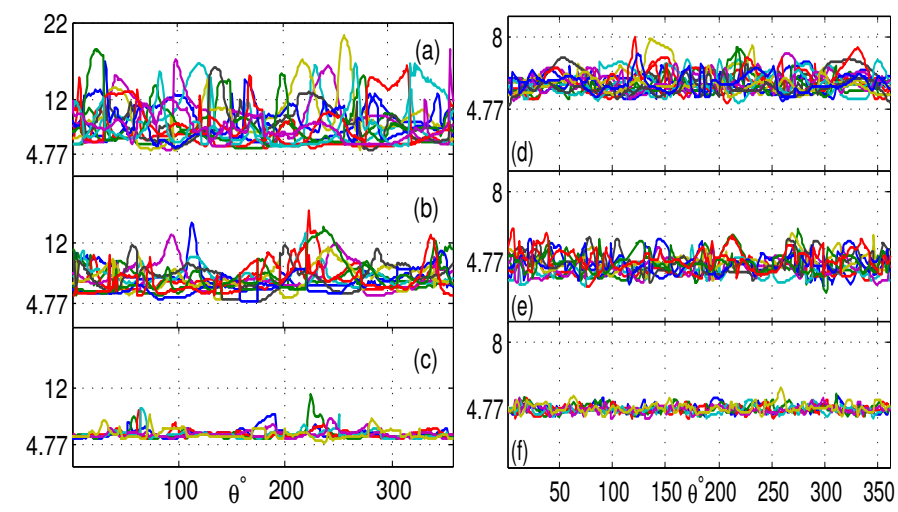

Fig. 8. Global $L_{\text {tot }}^{E}$ (in $\mathrm{dB}$ ) versus stirrer/hemisphere position (whole rotation with $1^{\circ}$ step) in conventional (a-c) and chaotic (d-f) cavities, (a)\&(d) 15 modes above $700 \mathrm{MHz}$, (b)\&(e) 10 modes above $800 \mathrm{MHz}$ and (c)\&(f) 6 modes above $1.2 \mathrm{GHz}$.

This study has been carried out on three frequency ranges above the $60^{\text {th }}$ mode while considering 15 modes above 700 $\mathrm{MHz}, 10$ modes above $800 \mathrm{MHz}$ and 6 modes above 1.2 GHz. As we already mentioned the similar behaviors of the indicators based on electric and magnetic fields, only the electric energy distribution will be considered here for 360 positions of the stirrer and of the hemisphere on a whole rotation (for a rotation angular step of $1^{\circ}$ ).

For each considered mode of the classical RC, Fig. 8 indicates the variation of the related global IPR indicator $L_{t o t}^{E}$ versus the stirrer (Fig.8a-c) or hemisphere position (Fig.8df). Its mean and standard deviation over all the stirrer $\backslash$ hemisphere angular positions are indicated in Fig.9. 
Fig. 9 shows a decrease with the frequency range of $L_{t o t}^{E}$ mean values over the stirrer or hemisphere rotation, corresponding to a weakening of the localization phenomenon. For the chaotic cavity, mean values close to the ideal value (3 in linear or $4.77 \mathrm{~dB}$ ) are obtained on the two highest frequency bands. The decrease of $L_{\text {tot }}^{E}$ standard deviations with the frequency is also obvious; thus fewer modes suffer from high localization effect. However, even if the same trends are observed for both cavities, on a given frequency band the performances of the chaotic cavity are always better than those of the classical RC, with mean values of $L_{t o t}^{E}$ closer to 3 and lower standard deviations. Thus, in the chaotic cavity, the eigenfield distributions are more uniform and less sensitive to the stirring object position than in the classical RC.

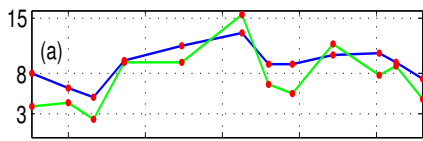

$\begin{array}{lllll}0.7065 & 0.7134 & 0.7203 & 0.7271 & 0.734\end{array}$
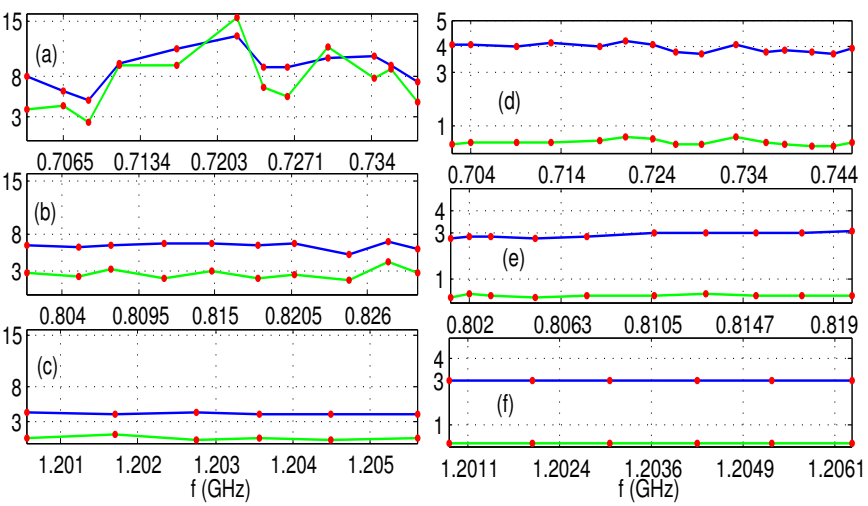

Fig. 9. Means (blue) and standard deviations (green) over stirrer/hemisphere rotation of the global $L_{t o t}^{E}$ (linear scale), in conventional (a-c) and chaotic (d-f) cavities, (a)\&(d) 15 modes above $700 \mathrm{MHz}$, (b)\&(e) 10 modes above $800 \mathrm{MHz}$ and (c)\&(f) 6 modes above $1.2 \mathrm{GHz}$. The values for each mode are indicated at its resonant frequency at the initial stirrer/hemisphere position.

From $L_{\text {tot }}^{E}$ mean values over the stirrer rotation calculated for the different modes of each frequency band, a mean value over the modes is calculated. In order to quantify the behavior differences between the modes of a given frequency band, the standard deviation of the same ensemble $L_{\text {tot }}^{E}$ mean values is also determined over the modes of each studied frequency band. The obtained means and standard deviations are given in Tab.III.

TABLE III

\begin{tabular}{|c|c|c|c|c|c|c|}
\hline & \multicolumn{2}{|c|}{$700 \mathrm{MHz}$} & \multicolumn{2}{|c|}{$800 \mathrm{MHz}$} & \multicolumn{2}{|c|}{$1.2 \mathrm{GHz}$} \\
\hline$\left\langle\left\langle L_{\text {tot }}^{E}\right\rangle_{\text {pos }}\right\rangle_{f}$ & $\mu$ & $\sigma$ & $\mu$ & $\sigma$ & $\mu$ & $\sigma$ \\
\hline $\mathrm{C} 1$ & 9.08 & 2.20 & 6.35 & 0.50 & 4.12 & 0.06 \\
\hline $\mathrm{C} 2$ & 3.95 & 0.15 & 3.82 & 0.08 & 3.00 & 0.03 \\
\hline
\end{tabular}

Means and standard deviations of $\left\langle L_{t o t}^{E}\right\rangle_{\text {pos }}$ on the three frequency bands.

These results highlight the better field uniformity in the chaotic cavity with mean values on the three frequency bands lower than the one attained on the highest frequency band in the conventional RC. Moreover, the much lower standard deviations indicate a better spectral uniformity obtained in this alternative RC. The improvement of the cavity properties while increasing the frequency is also noticeable with these results. In particular, the mean value attains the ideal one (namely 3 ) in the highest frequency band for the chaotic cavity.

\section{TOTAL FIELD PROPERTIES}

\section{A. Field reconstruction}

Uniformity and anisotropy coefficients have been defined in the norm [1] to be applied to measurement results. They are calculated from the electric field within the excited RC and not the previously examined eigenmodes. Before applying them in order to compare the performances of both cavities regarding standardized criteria, the excited field has to be rebuilt from the numerically determined eigenmodes. As the eigenmodes have been numerically determined without considering any losses, this approach is approximate but can be considered as valid for low losses.

For a cavity excited at the angular frequency $\omega$, the electric field at the same frequency can be expanded on the cavity eigenmodes as [19]:

$$
\vec{E}_{(\omega)}=-\frac{\jmath \omega \mu}{V} \sum_{m=1}^{\infty} \frac{1}{k_{m}^{2}-k^{2}}\left(\frac{\int_{V} \vec{J}\left(\overrightarrow{r_{0}}\right) \cdot \vec{e}_{m}\left(\overrightarrow{r_{0}}\right) d v}{\int_{V}\left|\vec{e}_{m}\right|^{2} d v}\right) \cdot \vec{e}_{m}
$$

where $k^{2}=k_{0}^{2}\left[1-(-1+\jmath) \cdot \omega_{m} /\left(Q_{m} \omega\right)\right]$, with $Q_{m}$ the quality factor of the $m^{t h}$ eigenmode. $\vec{e}_{m}$ is the $m^{t h}$ eigenmode field whose mean of the square electric field amplitude on the whole cavity volume $V$ is normalized to one. $k_{m}$ is related to the $m^{\text {th }}$ resonant angular frequency $\omega_{m}$ by $k_{m}=\omega_{m} / c$, with $c$ the speed of light in vacuum. The current $\vec{J}$ represents the source and $\overrightarrow{r_{0}}$ its location in the cavity.

The quality factors $Q_{m}$ are calculated by considering the effects of the Joule losses on the cavity walls and of the cavity loading by an antenna, according to $\left(Q_{m}\right)^{-1}=\left(Q_{m}^{\text {Joule }}\right)^{-1}+$ $\left(Q_{m}^{a n t}\right)^{-1}$. The $Q_{m}^{\text {Joule }}$ and $Q_{m}^{\text {ant }}$ are calculated in the high frequency approximation [20] [21] (Eq. 5).

$$
Q_{m}^{\text {Joule }}=\frac{3 V}{2 S \delta} \text { and } Q_{m}^{a n t}=16 \pi^{2} V\left(\frac{f}{c}\right)^{3}
$$

with $S$ the total surface area of the cavity walls and $\delta$ the wall skin depth.

In order to ensure the obtained results are not specific to a given excitation location, eight pointlike current sources are considered within both cavity volumes (Fig.3), these unit current sources having an amplitude of $1 / \sqrt{3}$ along each axis of the Cartesian coordinate system in order to excite similarly the three field components.

\section{B. Impact of the localization to the eigenmodes contributions}

The previous study of the eigenmodes properties showed that in the classical RC numerous modes have their energy highly concentrated around the stirrer to the detriment of the energy in the working area. According to Eq.4, the electric field within the working area is the sum of the contribution of each eigenmode; if an eigenfield is mostly concentrated around the stirrer, its contribution to the total field within the working volume will be reduced. To show the impact of the eigenfield localization outside the useful area on its contribution to the total field in the useful volume, we define the modal contribution $E_{m}$ of the $m^{t h}$ mode as the excited electric field obtained while considering only the $m^{\text {th }}$ mode, without the contribution of the other modes. Derived from 
Eq.4, the modal contribution $E_{m}$ of the $m^{t h}$ mode can be written :

$$
\vec{E}_{m\left(\omega_{m}\right)}=-\frac{\jmath \omega \mu}{V\left(k_{m}^{2}-k^{2}\right)}\left(\frac{\int_{V} \vec{J}\left(\overrightarrow{r_{0}}\right) \cdot \vec{e}_{m}\left(\overrightarrow{0_{0}}\right) d v}{\int_{V}\left|\vec{e}_{m}\right|^{2} d v}\right) \cdot \vec{e}_{m}
$$

and its amplitude is maximal at the $m^{t h}$ mode resonant angular frequency $\omega_{m}$.

From the extraction of the three electric field components of the $m^{\text {th }}$ eigenmode at the center of the working volume, we calculate the contribution at this central point of the first 10 eigenmodes above $700 \mathrm{MHz}$ (Fig.10.a for the conventional RC and Fig.10.c for the chaotic one) while considering the $S_{1}$ excitation location in both cavities. The total electric field due to the contribution of these 10 modes and calculated using Eq. 4 is presented in Fig.10.b for the conventional RC and Fig.10.d for the chaotic one.

In Fig.10.a, representing the evolution of the modal frequency weight $\left|E_{m}(\omega)\right|$ of 10 modes of the classical RC, one distinguishes only 6 peaks, 4 invisible peaks corresponding to maximum amplitudes $\left|E_{m}\left(\omega_{m}\right)\right|$ below $0.4 \mathrm{~V} / \mathrm{m}$ or $2.6 \%$ of the largest peak (of $15.2 \mathrm{~V} / \mathrm{m}$ ); these low weight modes weakly contribute to the total field and modal overlap (Fig.10.b). For the chaotic cavity in contrast, the lowest peak amplitude is of $1.34 \mathrm{~V} / \mathrm{m}$ or $8.1 \%$ of the largest peak (of $16.5 \mathrm{~V} / \mathrm{m}$ ). Thus all peaks are visible in Fig.10.c and the contribution of the 10 modes is found on the total field (Fig.10.d). The greatest disparity between the peak amplitudes for the different modes in the conventional cavity results in a normalized standard deviation between the amplitudes of 1.25 against 0.69 in the chaotic cavity.

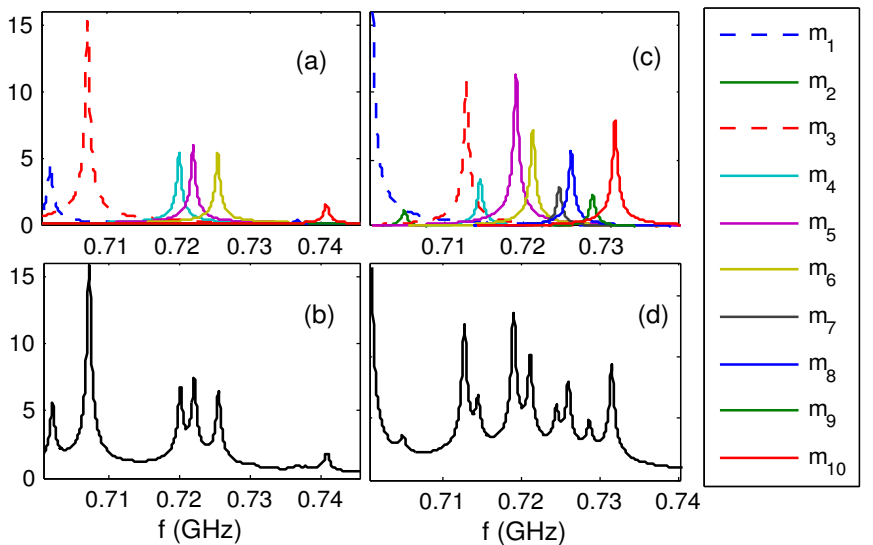

Fig. 10. Amplitude of the modal contribution $\left|E_{m(\omega)}\right|$ at the center of working volume for the eigenmodes $m_{i}$ ((a) for classical cavity and (c) for chaotic cavity); and amplitude of the total electric field induced by their contribution ((b) for classical cavity and (d) for chaotic cavity).

Let us now relate these modal contributions and localization indicators previously calculated. For the 10 modes considered, the mean values and standard deviations of $L_{t o t}^{E}$ are 14.6 and 17.12 in the conventional cavity against 3.95 and 0.25 in the chaotic cavity. Indeed, no mode presents a high energy localization in the chaotic cavity, contrary to the conventional cavity. In the latter, the most important localization effect is obtained for the $7^{\text {th }}$ mode $\left(R_{b}=3.05, L_{\text {tot }}^{E}=61.31\right)$ associated with an invisible amplitude peak $(0.147 \mathrm{~V} / \mathrm{m})$. In contrast, the highest amplitude peak of the modal contributions $(15.2 \mathrm{~V} / \mathrm{m}$ for mode 3$)$ is associated with parameters $\left(R_{b}=0.43, L_{t o t}^{E}=\right.$ $4.34)$ indicating no energy localization around the stirrer. This shows the negative impact of energy localization patterns on spectral uniformity of the total field and on the modal overlap, the latter playing an important role in obtaining a uniform and homogeneous field in the RC [22].

\section{Field homogeneity and isotropy}

According to Eq.4, the spectral bandwidth of each resonance frequency is non zero. As a consequence, the eigenmodes are not only excited at their resonance frequency, and even if their weight rapidly decreases outside the resonance width, modal overlap occurs. It implies that the properties of the excited field are not strictly equivalent to those of the isolated eigenmodes, and that the rebuilt field of Eq.4 has to be used to apply uniformity and anisotropy standardized criteria. In the following, the rebuilt field is calculated for frequency steps chosen equal to the third of the smallest frequential mode bandwidth in each frequency band, respectively of $14.67 \mathrm{kHz}$, $12.22 \mathrm{kHz}$ and $5.91 \mathrm{kHz}$ for the chaotic cavity and 13.74 $\mathrm{kHz}, 11.01 \mathrm{kHz}$ and $5.45 \mathrm{kHz}$ for the classical RC.

To characterize the field uniformity, four standard deviations are used. Three of them, associated to the orthogonal field components $E_{i}(i=x, y$ or $z$ in our case), are calculated as [1]

$$
\sigma_{i, d B}=20 \cdot \log \left(1+\frac{\sigma_{i}}{\left\langle E_{i \max }\right\rangle}\right)
$$

where the standard deviation $\sigma_{i}$ and mean value $\left\langle E_{i \max }\right\rangle$ are calculated from the maximal field amplitudes attained over a stirrer or hemisphere rotation at each of the eight vertices of the working volume, the latter one being chosen to present the same volume in both cavities. A global uniformity indicator $\sigma_{\text {tot }}$ is also calculated using the maximal field amplitudes obtained by considering the three field components. Only independent stirrer/hemisphere positions are considered, i. e. 20, 22 and 32 positions on the three frequency bands for the chaotic cavity, and 18, 20 and 25 positions in the classical one, as indicated by correlation coefficients between normalized eigenfields.

Fig. 11 shows the four standard deviations obtained in three bandwidths for both cavities while considering the excitation $S_{1}$ (indicated in Fig.3). The standard deviations are lower in the chaotic cavity and seldom reach $3 \mathrm{~dB}$. According to the norm, the total standard deviation has to be below $3 \mathrm{~dB}$ with a tolerance of a maximum of three peaks above $3 \mathrm{~dB}$ per octave. In the chaotic cavity, the standard deviations stay below the $3 \mathrm{~dB}$ limit in the two higher frequency bands, whereas it is only respected in the highest band in the classical RC.

The frequential averages of the four curves of Fig.11 are given in Tab.IV. They highlight the lower standard deviations in the chaotic cavity, as all the average values are lower in this cavity; it indicates that a better field uniformity is obtained. We also notice in this cavity lower differences between the mean standard deviations related to the three field components; it shows a better field isotropy. These mean values also confirm the decrease of the standard deviations with the frequency, but the properties of $\mathrm{C} 2$ always stay better than those of $\mathrm{C} 1$. 

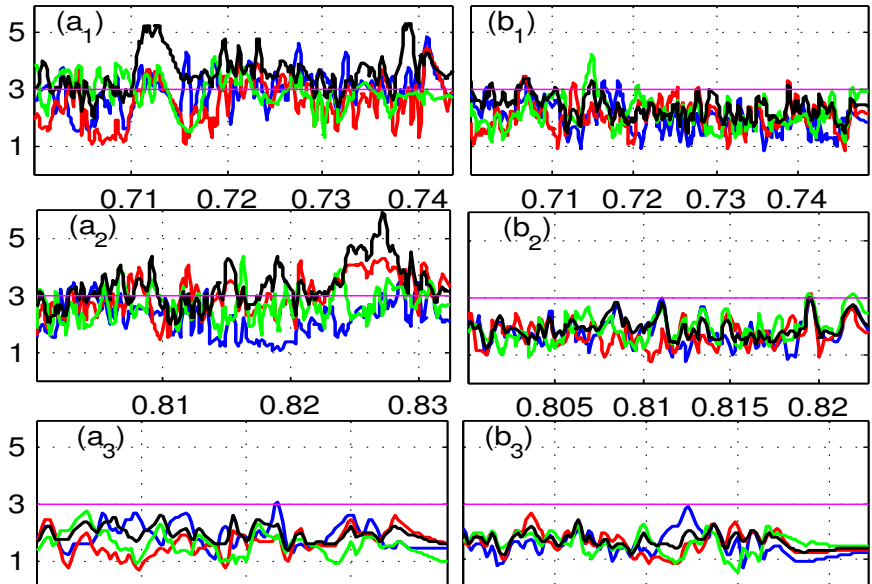

1.2021 .2041 .206

f $(\mathrm{GHz})$
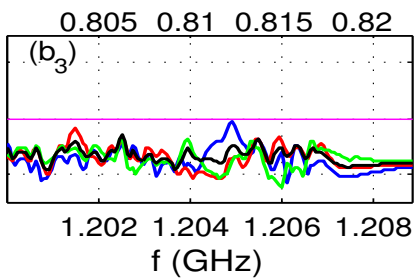

Fig. 11. Standard deviations of the three Cartesian field components and total one (blue for $\sigma_{x}$, red for $\sigma_{y}$, green for $\sigma_{z}$, and black for $\sigma_{t o t}$ ) for $\left(a_{1}, a_{2}, a_{3}\right)$ cavity $\mathrm{C}_{1}$ and $\left(b_{1}, b_{2}, b_{3}\right)$ cavity $\mathrm{C}_{2}$ in the three frequency bands for a source located in $\left(S_{1}\right)$.

TABLE IV

\begin{tabular}{|l|l|l|l|l|l|l|}
\cline { 3 - 7 } \multicolumn{2}{c|}{} & $\left\langle\sigma_{x}\right\rangle$ & $\left\langle\sigma_{y}\right\rangle$ & $\left\langle\sigma_{z}\right\rangle$ & $\left\langle\sigma_{\text {tot }}\right\rangle$ & $\delta \sigma_{k}$ \\
\hline \multirow{2}{*}{$700 \mathrm{MHz}$} & $\mathrm{C} 1$ & 2.99 & 2.57 & 2.90 & 3.61 & 0.42 \\
\cline { 2 - 8 } & $\mathrm{C} 2$ & 1.98 & 2.05 & 2.15 & 2.37 & 0.17 \\
\hline \multirow{2}{*}{$800 \mathrm{MHz}$} & $\mathrm{C} 1$ & 2.18 & 2.99 & 2.64 & 3.32 & 0.81 \\
\cline { 2 - 7 } & $\mathrm{C} 2$ & 1.76 & 1.69 & 1.89 & 1.90 & 0.20 \\
\hline \multirow{2}{*}{$1200 \mathrm{MHz}$} & $\mathrm{C} 1$ & 1.90 & 1.63 & 1.58 & 1.94 & 0.32 \\
\cline { 2 - 7 } & $\mathrm{C} 2$ & 1.48 & 1.57 & 1.54 & 1.63 & 0.09 \\
\hline
\end{tabular}

Frequency means on the three frequency bands of the four standard deviations and maximal difference between the three ones related to field components for both cavities, source at $S_{1}$.

Using the same procedure for the field reconstruction at the eight vertices of the working volume and the stirring process, we also determined the field anisotropy coefficients $\left(A_{\alpha \beta}\right)$ given by [1]:

$$
A_{\alpha \beta}=\frac{\left|E_{\alpha}\right|^{2}-\left|E_{\beta}\right|^{2}}{\left|E_{\alpha}\right|^{2}+\left|E_{\beta}\right|^{2}}
$$

where $\alpha$ and $\beta$ indicate directions of mutually orthogonal Cartesian components, and the average is performed firstly on the hemipshere or stirrer positions then on the eight field extraction points. From these three partial anisotropy coefficients, a global anisotropy indicator $A_{t o t}$ can also be calculated using :

$$
A_{t o t}=\sqrt{\left[A_{x y}^{2}+A_{y z}^{2}+A_{z x}^{2}\right] / 3}
$$

Fig.12 shows the amplitude of these four electric field anisotropy coefficients on the three frequency bands in both cavities.

First we note that the anisotropy coefficients decrease with frequency in both cavities. In all frequency bands, the anisotropy coefficients are lower in the chaotic cavity, indicating a better electric field isotropy. According to the standard [1], with a number of independent positions between 10 and 30 as in our case, a good stirring quality is characterized by a total anisotropy coefficient less than or equal to $-5 \mathrm{~dB}$, whereas a medium stirring quality is associated to the limit $-2.5 \mathrm{~dB}$. In the chaotic cavity, the total anisotropy coefficient remains lower than $-2.5 \mathrm{~dB}$ in the three frequency bands and decreases with frequency, whereas in the cavity with a stirrer

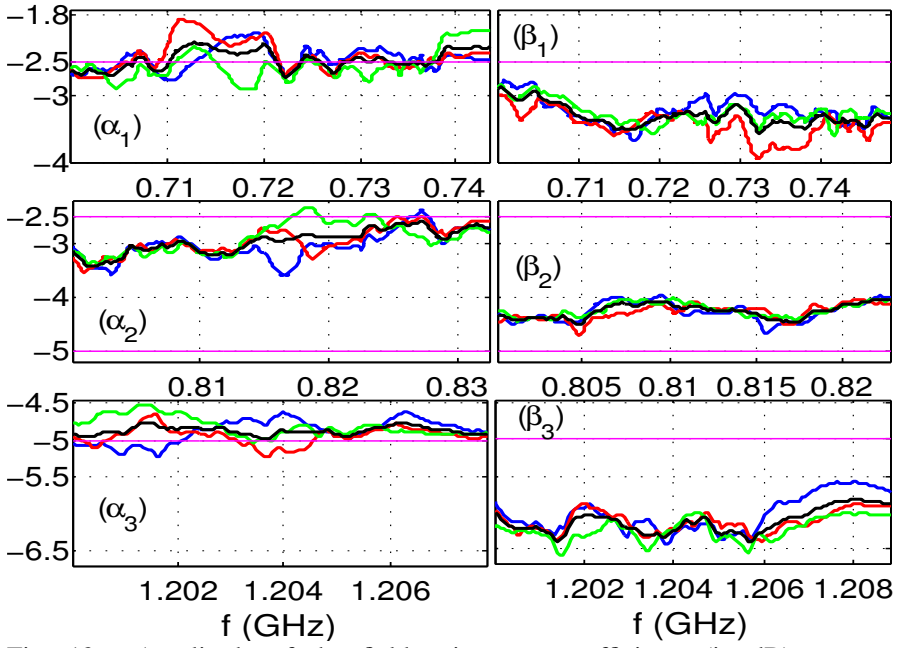

Fig. 12. Amplitude of the field anisotropy coefficients (in dB) versus frequency (blue for $A_{x y}$, red for $A_{y z}$, green for $A_{z x}$, and black for $A_{t o t}$ ) for $\left(\alpha_{1}, \alpha_{2}, \alpha_{3}\right)$ cavity $\mathrm{C}_{1}$ and $\left(\beta_{1}, \beta_{2}, \beta_{3}\right)$ cavity $\mathrm{C}_{2}$, source location $\left(S_{1}\right)$ in the three frequency bands.

this criterion is only respected in the band above $1.2 \mathrm{GHz}$. However, the standard is respected below the $-2.5 \mathrm{~dB}$ limit, and this is achieved on the highest frequency band for both cavities. Based on these values and according to the norm [1], the chaotic cavity permits to obtain a good stirring quality (total anisotropy coefficient below $-5 \mathrm{~dB}$ ) whereas the conventionnal $\mathrm{RC}$ can only provide a medium stirring quality.

To confirm these remarks, the amplitudes of the four anisotropy coefficients have been averaged on each frequency band (Tab.V). We notice the decrease of these values with the frequency, and the best performances of the chaotic cavity, with lower mean values.

TABLE V

\begin{tabular}{|l|l|l|l|l|l|}
\cline { 3 - 6 } \multicolumn{2}{c|}{} & $\left\langle A_{x y}\right\rangle$ & $\left\langle A_{y z}\right\rangle$ & $\left\langle A_{z x}\right\rangle$ & $\left\langle A_{\text {tot }}\right\rangle$ \\
\hline \multirow{2}{*}{$700 \mathrm{MHz}$} & $\mathrm{C} 1$ & -2.44 & -2.40 & -2.54 & -2.67 \\
\cline { 2 - 6 } & $\mathrm{C} 2$ & -3.23 & -3.44 & -3.26 & -3.31 \\
\hline \multirow{2}{*}{$800 \mathrm{MHz}$} & $\mathrm{C} 1$ & -3.05 & -2.96 & -2.90 & -2.97 \\
\cline { 2 - 6 } & $\mathrm{C} 2$ & -4.28 & -4.29 & -4.24 & -4.27 \\
\hline \multirow{2}{*}{$1200 \mathrm{MHz} z$} & $\mathrm{C} 1$ & -4.88 & -4.94 & -4.81 & -4.88 \\
\cline { 2 - 6 } & $\mathrm{C} 2$ & -6.03 & -6.13 & -6.24 & -6.13 \\
\hline
\end{tabular}

Frequency means of anisotropy coefficient amplitudes (in dB), source at $S_{1}$.

We have shown that, for a point like source located at $S_{1}$, the electric field over a stirrer or hemisphere rotation is more uniform and isotropic in the chaotic cavity. We will now verify that this conclusion is independent of the excitation location.

Tab.VI shows the frequency means on the three frequency bands of the total standard deviation $\sigma_{\text {tot }}$ and the total anisotropy coefficient $A_{t o t}$ for the seven other source locations $S_{i}$ presented in Fig.3. Similar results are obtained at all the source locations, and the conclusions drawn at the first source location remain valid: the electric field is always more uniform and isotropic in the chaotic cavity and its properties improve in both cavities with frequency increasing. The latter results allow us to confirm the better properties of the chaotic cavity regardless of the source position.

\section{CONClusion}

It has been demonstrated that an energy localization phenomenon appears around the mode stirrer of a classical RC 
TABLE VI

\begin{tabular}{|c|c|c|c|c|c|c|c|}
\hline & \multicolumn{2}{|c|}{$700 \mathrm{MHz}$} & \multicolumn{2}{|c|}{$800 \mathrm{MHz}$} & \multicolumn{2}{|c|}{$1200 \mathrm{MHz}$} \\
\hline & & $\mathrm{C} 1$ & $\mathrm{C} 2$ & $\mathrm{C} 1$ & $\mathrm{C} 2$ & $\mathrm{C} 1$ & $\mathrm{C} 2$ \\
\hline \multirow{2}{*}{$S_{2}$} & $\left\langle\sigma_{t o t}\right\rangle$ & 3.693 & 2.209 & 3.344 & 1.837 & 2.448 & 1.651 \\
\hline & $\left\langle A_{t o t}\right\rangle$ & -2.323 & -3.261 & -3.065 & -4.280 & -4.693 & -6.051 \\
\hline \multirow[b]{2}{*}{$S_{3}$} & $\left\langle\sigma_{t o t}\right\rangle$ & 3.315 & 2.251 & 3.246 & 1.983 & 2.107 & 1.662 \\
\hline & $\left\langle A_{t o t}\right\rangle$ & -2.525 & -3.286 & -3.047 & -4.253 & -4.828 & -6.089 \\
\hline \multirow{2}{*}{$S_{4}$} & $\left\langle\sigma_{t o t}\right\rangle$ & 3.744 & 2.255 & 3.158 & 1.881 & 2.074 & 1.675 \\
\hline & $\left\langle A_{t o t}\right\rangle$ & -2.459 & -3.262 & -3.071 & -4.300 & -4.872 & -6.068 \\
\hline \multirow{2}{*}{$S_{5}$} & $\left\langle\sigma_{t o t}\right\rangle$ & 3.386 & 2.218 & 3.150 & 1.901 & 2.215 & 1.562 \\
\hline & $\left\langle A_{t o t}\right\rangle$ & -2.601 & -3.281 & -3.168 & -4.302 & -4.852 & -6.033 \\
\hline \multirow{2}{*}{$S_{6}$} & $\left\langle\sigma_{t o t}\right\rangle$ & 3.501 & 2.344 & 3.221 & 1.994 & 2.044 & 1.611 \\
\hline & $\left\langle A_{t o t}\right\rangle$ & -2.496 & -3.275 & -3.102 & -4.250 & -4.793 & -6.033 \\
\hline \multirow{2}{*}{$S_{7}$} & $\left\langle\sigma_{t o t}\right\rangle$ & 3.451 & 2.269 & 3.265 & 1.889 & 1.929 & 1.566 \\
\hline & $\left\langle A_{t o t}\right\rangle$ & -2.410 & -3.262 & -3.019 & -4.291 & -4.834 & -6.072 \\
\hline \multirow{2}{*}{$S_{8}$} & $\left\langle\sigma_{t o t}\right\rangle$ & 3.608 & 2.396 & 3.134 & 1.986 & 2.125 & 1.604 \\
\hline & $\left\langle A_{\text {tot }}\right\rangle$ & -2.462 & -3.269 & -3.067 & -4.251 & -4.804 & -6.052 \\
\hline
\end{tabular}

even at high frequencies. As the resonant modes undergoing this localization weakly contribute to the total field within the working volume, it degrades the spatial and spectral properties of the field in this useful area. To overcome this problem, a new RC shape has been proposed, that has been obtained through simple modifications of the conventional RC. The localization phenomenon is avoided in this case, so that the energy distribution is more uniform within the whole cavity and the modes contribution to the field within the working volume is stronger and less frequency dependent. The resulting improvements of the field properties are clear by considering standard uniformity and isotropy criteria, as the well operating indicators move below the required threshold at lower frequency and, on a given frequency band, always indicate a better operating of the chaotic cavity. The proposed simple and low-cost modifications of classical RC could thus permit to improve the field properties within a classical RC and also to decrease its Lowest Useable Frequency.

\section{REFERENCES}

[1] IEC 61000-4-21: Electromagnetic Compatibility (EMC)Part 4-21: Testing and Measurement TechniquesReverberation chamber test methods, International Electrotechnical Commission, IEC SC77B-CIS PR/A JWG REV, Geneva, Switzerland, Aug. 2003.

[2] S.W. McDonald and A.N. Kaufman, "Wave chaos in the stadium: statistical properties of short-wave solutions of the Helmholtz equation", Phys. Rev. A, 1988, vol. 37, p. 3067.

[3] E.J. Heller, "Bound-state eigenfunctions of classically chaotic Hamiltonian systems: scars of periodic orbits", Phys. Rev. Lett., 1984, vol. 53, no. 16, p. 1515.

[4] E.B. Bogomolny, "Smoothed wave functions of chaotic quantum systems", Physica D: Nonlinear Phenomena, 1988, vol. 31, no 2, p. 169-189.

[5] S.W. Kim, H.W. Lee, "Quantum-classical correspondence in localization of eigenstates for a system having mixedtype classical dynamics", Phys. Rev. E, 1999, vol. 59, no 5, p. 5384.
[6] L. Kaplan, E. Heller, "Short-time effects on eigenstate structure in Sinai billiards and related systems", Phys. Rev. $E$, 2000, vol. 62, no 1, p. 409.

[7] E. Damborsky, L. Kaplan, "Scar intensity statistics in the position representation”, Phys. Rev. E, 2005, vol. 72, no 6, p. 066204.

[8] P. Pradhan, S. Sridhar, "Correlations due to localization in quantum eigenfunctions of disordered microwave cavities". Physical review letters, 2000, vol. 85, no 11, p. 2360.

[9] J.-B. Gros, O. Legrand, F. Mortessagne, E. Richalot, K. Selemani, "Universal behaviour of a wave chaos based electromagnetic reverberation chamber", Wave Motion (2013).

[10] G. Orjubin, E. Richalot, S. Mengue, M.-F. Wong, and O. Picon. "On the FEM modal approach for a reverberation chamber analysis". Electromagnetic Compatibility, IEEE Transactions., on, 49(1) p :76-85, 2007.

[11] H.J. Stöckmann, Chaos: an introduction, Cambridge University Press, 1999.

[12] R.A. Méndez-Sànchez, U. Kuhl, M. Barth, C. H. Lewenkopf, and H.-J. Stöckmann, "Distribution of Reflection Coefficients in Absorbing Chaotic Microwave Cavities", Phys. Rev. Lett., vol. 91, no. 17, Oct. 2003.

[13] M.L. Mehta, Random Matrices, Elsevier, 2004.

[14] K. Selemani, J.-B. Gros, E. Richalot, O. Legrand, O. Picon, F. Mortessagne, "Comparison of reverberation chamber shapes inspired from chaotic cavities", IEEE Trans. Electromag. Compat., vol. 57, no. 1, pp. 3-11, Feb. 2015.

[15] E. Richalot, S. Mostarshedi, O. Picon, K. Selemani, O. Legrand, F. Mortessagne, J.B. Gros, B. Sudret, J. Wiart, "Increasing contribution of stochastic methods in the characterization of complex systems: reverberation chamber and urban environment", ICEAA, 9-13 September 2013.

[16] F. Wegner, "Inverse Participation Ratio in $2+\varepsilon$ Dimensions", Zeitschrift für Physik B Condensed Matter, Vol. 36, 1980, pp. 209-214.

[17] Ya. V. Fyodorov, A. D. Mirlin, "Statistical properties of eigenfunctions in a disordered metallic sample", JETP Lett. Vol. 60, No. 11, pp. 790-790, Dec. 1994.

[18] P. Pradhan, S. Sridhar, "From chaos to disorder: Statistics of the eigenfunctions of microwave cavities", J. Phys., Vol. 58, No. 2, Feb. 2002.

[19] G. Cerri, V. M. Primiani, S. Pennesi, and P. Russo, "Source stirring mode for reverberation chambers", IEEE Trans. Electromagn. Compat., vol. 47, no. 4, pp. 815-823, Nov. 2005.

[20] J.M. Dunn, "Local, High-Frequency Analysis of the Fields in a Mode-Stirred Chamber", IEEE Trans. Electromagnetic Compatibility, Vol. 32, NO. 1, Feb. 1990, pp. 53-58.

[21] A. K. Mitra and T. F. Trost, "Power Transfer Characteristics of a Microwave Reverberation Chamber", IEEE Trans. Electromagnetic Compatibility, Vol. 38, NO. 2, May 1996, pp. 197-200.

[22] G. Gradoni and L.R. Arnaut. "Generalized extreme-value distributions of power near a boundary inside electromagnetic reverberation chambers". Electromagnetic Compatibility, IEEE Transactions on, 52(3) :506-515, 2010. 


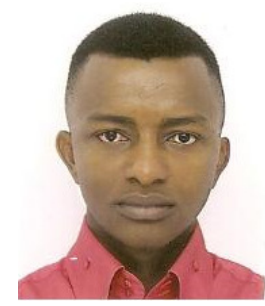

Kamardine SELEMANI was born in the Comoros in 1986. He received the M.Sc. degree in High frequency communication systems and the Ph.D. degree in telecommunications and electronics from the University of Paris-Est Marne-la-Vallée, Champssur-Marne, France, in 2010 and 2014, respectively. He has been a Graduate Teaching/Research Assistant with this University then with the ENSEA, CergyPontoise, France, before joining the CMN group, Cherbourg, France. His current research interests include EMC and electromagnetic propagation.

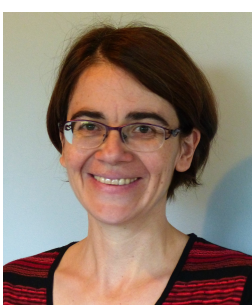

Elodie RICHALOT received the Diploma and $\mathrm{Ph} . \mathrm{D}$. degrees in electronics engineering from ENSEEIHT school in Toulouse, France, in 1995 and 1998, respectively. Since 1998, she has been with the University of Paris-Est Marne-la-Vallée, Champssur-Marne, France, where she became a Professor in electronics in 2010.

Her current research activities in ESYCOM laboratory include modeling techniques, electromagnetic compatibility and reverberantion chambers, and millimeter wave transmission lines.

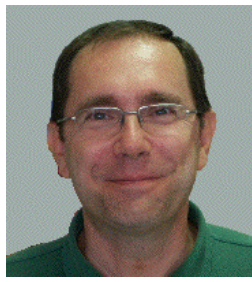

Olivier LEGRAND was born in France in 1961. $\mathrm{He}$ received the Ph.D. degree in theoretical physics from the Université d'Aix-Marseille II, Marseille, France, in 1987, and the Habilitation degree from the Université de Nice-Sophia Antipolis, Nice, France, in 1998.

In 1990, he joined the Université de Nice-Sophia Antipolis, where, since 2001, he has been a Professor of physics. His current research interests include linear and nonlinear waves, wave chaos, and wave propagation in complex media.

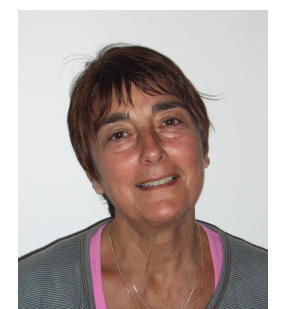

Odile PICON (M88) was born in Paris, France She received the "Agregation de Physique", prepared at the "Ecole Normale Supérieure de Fontenay aux Roses" (1976), the Doctor Degree in external geophysics from the University of Orsay, France (1980) and the Doctor in Physics Degree in Microwave CAD from the University of Rennes, France (1988) She was a teacher from 76 to 82 . Then, she acted as a Research Engineer in the "Space and Radioelectric Transmission" division of the "Centre National d'Etudes des Télécommunications" (CNET), from 82 to 91 . Since 91, she is a Professor of Electrical Engineering, first at the Paris7-University, then at the Université Paris-Est Marne-la-Vallée, France, where she has been heading the ESYCOM Laboratory. Her research work deals with electromagnetic theory, numerical methods for solving field problems, and design of millimeter wave passive devices. She has published more than 150 technical papers in books, journals and conferences.

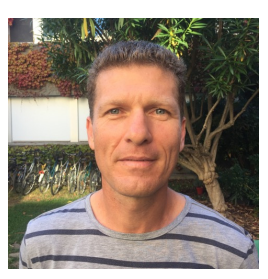

Fabrice MORTESSAGNE is currently a Physicist, a Professor at the University Nice Sophia Antipolis (UNS), and the Director of Laboratoire de Physique de la Matière Condenseé (UNS and CNRS).

His research activities include field of wave propagation in complex media and mesoscopic physics. His current works include chaotic scattering experiments in optics and microwaves, and microwave realizations of artificial graphene and other relativistic meta-materials. 